\title{
Transient decay of satellite lines of bound excitons in $\mathrm{Si}: \mathbf{P}$
}

\author{
A. T. Hunter, S. A. Lyon, ${ }^{*}$ D. L. Smith, and T. C. McGill \\ California Institute of Technology, Pasadena, California 91125
}

(Received 29 January 1979)

\begin{abstract}
The transient decay of the $\alpha$ and $\beta$ series of lines in the photoluminescence spectrum of Si:P are reported. The decays of the $\alpha$ lines are compared with the decays of the $\beta$ lines. The results are consistent with $\beta_{2}$ having the same initial state as $\alpha_{3}$. The results for $\beta_{1}$ and $\beta_{3}$ are less certain. Their decays are consistent with $\beta_{1}$ having the same initial state as either $\alpha_{2}$ or $\alpha_{3}$, and with $\beta_{3}$ having the same initial states as $\alpha_{4}$. Overlap between lines, uncertainty in the decay of the boron bound exciton and the presence of unidentified background all make it difficult to make conclusive line assignments.
\end{abstract}

\section{INTRODUCTION}

The satellite lines near the bound-exciton luminescence in doped $\mathrm{Si}$ have been the subject of rather extensive experimental ${ }^{1-13}$ and theoretical investigation. $^{4,14,15}$ For the donors, ${ }^{7,8,13}$ a set of lines are observed in both the no-phonon and phonon-assisted transitions (labeled the $\alpha$ series $^{7,8}$ ) and an additional set of lines (labeled the $\beta$ series $^{7,8}$ ) are observed in the phonon-assisted transitions. These lines are shown in Fig. 1. Both the $\alpha$ and $\beta$ series have been interpreted in terms of a shell model in which electrons fill the original valley-orbit split levels of the donor. ${ }^{15,16}$ The $\alpha$ series of lines are assumed to result from recombination of an electron from the $\Gamma_{1}$ shell with a hole. ${ }^{17}$ The $\beta$ series are interpreted as due to the recombination of an electron in the $\Gamma_{3,5}$ shell with a hole and, hence, are ground state to ground
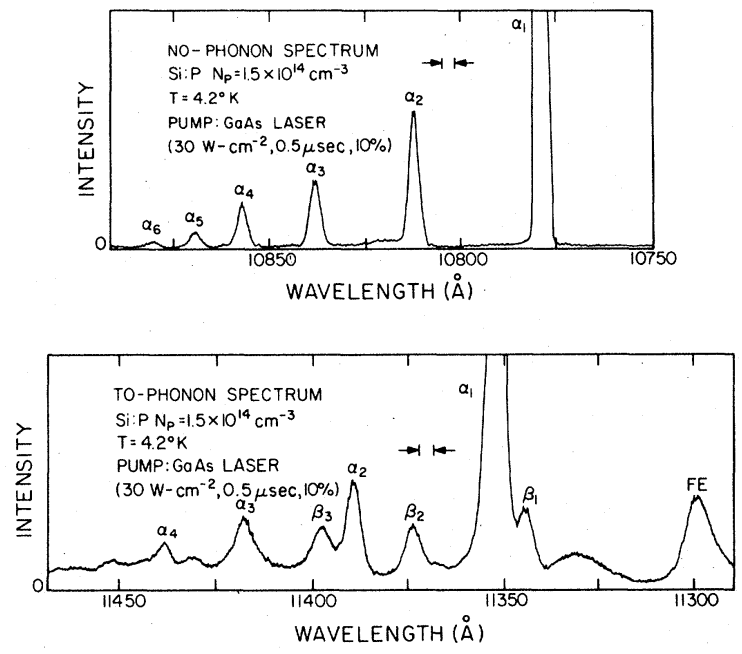

FIG. 1. Photoluminescence spectrum of $\mathrm{Si}: \mathrm{P}$. The upper spectrum covers the no-phonon replica energy range. The lower curve covers the transverse- and longitudinal-opticalphonon replica energy range. state transitions. This interpretation has had some successes in explaining the spectroscopic data. ${ }^{18}$ However, the systematics of the splitting of these lines for different donors is not what is qualitatively expected. ${ }^{13}$ Further, recent stress data show that two of the $\beta$ series $\left(\beta_{1}\right.$ and $\left.\beta_{2}\right)$ split into more lines than expected on the basis of the shell model. ${ }^{12}$

In the shell model the $\alpha_{n}$ line (we use the notation given in Ref. 7) should have the same initial state as the $\beta_{n-1}$ line and both should involve the binding of $n$ excitons. This prediction can be tested by examining the decay characteristics since lines with the same initial state should exhibit identical decays. Sauer, et al. ${ }^{9}$ have attempted to test this hypothesis by measuring the decay in the transverse-optical (TO) phonon-assisted transitions. They find that the decays of the $\alpha_{3}$ and $\beta_{2}$ differ as do the $\alpha_{4}$ and $\beta_{3}$. Hence, they conclude that the shell model interpretation is invalid. As shown in the upper spectrum in Fig. 1, the lines in the no-phonon spectra are sharp and distinct with little background, making possible an accurate measurement of the decay of a given line. However, as pointed out by Thewalt, ${ }^{10}$ there are more lines in the transverse-optical phonon-assisted transition (see lower spectrum in Fig. 1), the lines are broader, and the lines may be superimposed on a background. Hence, the determination of the decay characteristics using the TO phonon-assisted transitions may have been confused by the overlap of lines and background with different decay characteristics.

To attempt to determine which lines have the same initial states, we have made transient measurements on lightly doped $\mathrm{Si}: \mathrm{P}$ samples and analyzed the data taking account of the overlap of lines. The data were recorded to more accuracy at longer times than in Ref. 9. The decay of the $\alpha_{1} ; \alpha_{2}, \alpha_{3}$, and $\alpha_{4}$ lines were measured in the no-phonon (NP) replica. The decays of $\beta_{1}, \beta_{2}$, and $\beta_{3}$ were measured in the TOphonon replica since they do not occur in the nophonon region. The decay of the $\beta$ lines was compared with various combinations of the decays of the 
$\alpha$ lines as observed in the NP transition to assess which lines could have the same initial states.

This paper is organized in the following way: Section II describes the experimental setup and the steps taken to insure proper treatment of the dark count and response of the photon counting equipment. The experimental results are presented in Sec. III. Section IV includes the results from fitting the decay of the $\beta$ lines to various combinations of the $\alpha$ lines and our conclusions based on the quality of the various fits.

\section{EXPERIMENT}

The transient measurements were made on a 2 $\mathrm{mm}$ thick wafer of high-purity Waker silicon, transmutation doped with phosphorous to the $1.5 \times 10^{14} \mathrm{~cm}^{-3}$ level. Hall measurements indicated $N_{A}-N_{D}$ was $\sim 2 \times 10^{11} \mathrm{~cm}^{-3}$ before doping.

Luminescence measurements on the undoped sample did not show a detectable phosphorous line, but did show some boron bound-exciton (BE) luminescence. ${ }^{19}$ The relationship between BE luminescence intensity and concentration ${ }^{20}$ indicated the sample was not closely compensated and, therefore, very low in boron. The low boron content allowed a low boron to phosphorus ratio in the final, doped sample, while still maintaining a low enough phosphorus content to avoid concentration broadening. The presence of boron was undesirable because the boron $\mathrm{BE}$ line overlaps the phosphorous $\beta_{1}$ line.

The system used to generate and detect the luminescence signal was fairly straightforward, and will be described briefly. The sample and an RCA GaAs diode laser, used to pump the sample, were immersed in liquid $\mathrm{He}$ at $4.2^{\circ} \mathrm{K}$ in a Janis research Dewar. The laser light (centered at $8500 \AA$ ) was directed onto the center of a polished and etched surface of the sample, with a spot size of about $1 \mathrm{~mm}^{2}$. The peak excitation power density was varied between $\sim 8 \mathrm{~W} \mathrm{~cm}^{-2}$ and $\sim 70 \mathrm{~W} \mathrm{~cm}^{-2}$, by adjusting the amplitude of the current pulse driving the laser. Current pulses were of $0.5 \mu$ sec duration $5 \mu \mathrm{sec}$ apart. The luminescence from the edge of the sample was focused onto the aperture of a SPEX 1269 spectrometer. This sample geometry (pumping the flat surface of the wafer, and pointing the edge toward the spectrometer) was chosen because it maximized the signal. The resolution of the spectrometer was $3.5 \AA$ with the slit width and grating used for the experiment. A visible light filter placed in front of the aperture reduced stray light from the laser and the instrument-rack lamps. A liquid-nitrogen-cooled photomultiplier tube (PMT) detected the luminescence passing through the spectrometer. Finally, the output of the PMT was fed into an amplifier discriminator.
The luminescence signal leaving the amplifier discriminator was further processed by a system that measured and stored the time with respect to the laser pulse that the signal arrived. The timing was carried out with a time-to-amplitude converter (TAC); the results were stored in a multichannel analyzer (MCA). Additional electronics prevented the MCA from processing the TAC output if two or more photons were detected during the timing interval associated with a given laser pulse. The system could measure the time of arrival of only the first of the photons, and storing its time while ignoring that of the second photon would bias the data.

The response of the PMT was an important factor in the experiment, and deserves further discussion. The two nonideal features of the tube that affected our results were dark count and afterpulsing. The dark count gave a uniform (in time) background of approximately 6 counts/sec to all the data. Afterpulsing of the PMT was also a source of background in the transient measurements, but was not uniform in time. However, the nonuniformity proved to be negligible in this experiment. The transient curves stored on the MCA showed background both before the laser went on and after luminescence died away. Background was calculated by averaging over 50 channels $(\sim 350 \mathrm{nsec})$ in each of the regions. The two numbers agreed to within statistical error except for long-lived lines where the luminescence did not die away fast enough. Therefore, the PMT response was "deconvolved" from the signal merely by subtracting the average background from each channel of the MCA.

\section{EXPERIMENTAL DATA}

Using the system described above, various transients were measured. The lines measured in this way include the NP $\alpha$ series, the TO phonon $\beta_{1}, \beta_{2}$, and $\beta_{3}$. Pump power dependences of several lines were also investigated. Figure 2 illustrates two factors that determine, in part, the resolution and accuracy of the measurements. The first is the laser decay, which is quite fast in comparison to the luminescence decays. The second is the scatter in the data points. The size of the error bars (shown for several points) primarily reflects statistical uncertainty in the counts per channel before subtracting dark count and afterpulsing. The subtraction does not add much to the absolute uncertainty; it only increases the relative uncertainty.

Decays of $\alpha_{1-4}$, each at the same pump power, are shown in Fig. 2. The curves are exponential except in the earlier region, just after the laser shuts off. Decay times, derived from the later exponential parts of the curve, are $245 \mathrm{nsec}$ for $\alpha_{1}, 134 \mathrm{nsec}$ for $\alpha_{2}, 95$ nsec for $\alpha_{3}$, and $85 \mathrm{nsec}$ for $\alpha_{4}$. The error bars are 


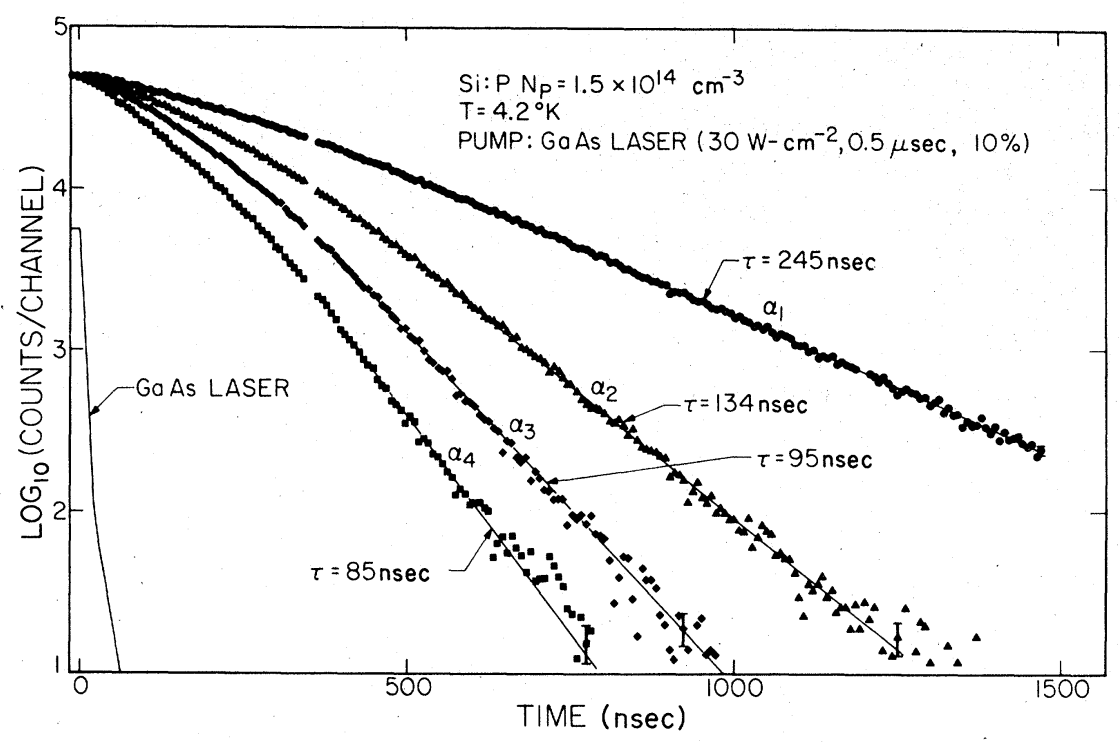

FIG. 2. Logarithm to base ten of the transient decay of the $\alpha_{1}, \alpha_{2}, \alpha_{3}$, and $\alpha_{4}$ lines in the no-phonon replica. The points are the experimental data. The solid lines are straight-line fits to the decays at long times. The decay times given are obtained by converting the slopes of these lines to base $e$. The solid line labeled $\mathrm{GaAs}$ laser is the experimental decay for the laser used in the experiments.

$\pm 10 \mathrm{nsec}$ for the $\alpha_{1}$ time, and \pm 5 for the other three. $\alpha_{5}$ and $\alpha_{6}$ show similar decays, except the curves break to slower decays at long times. The break is probably due to the presence of low intensity, longlived background lines, which show up in the $\alpha_{5}$ and $\alpha_{6}$ decays because both these lines are themselves low in intensity.
The rounding seen in all the $\alpha$ lines at early times increases with pump power, as illustrated for $\alpha_{3}$ in Fig. 3. The decays all become exponential eventually, with the same lifetime, but for the highest pump power the curve does not become straight until over $600 \mathrm{nsec}$ after the laser has shut off. This initial rounding would lengthen "decay times" derived by fit-

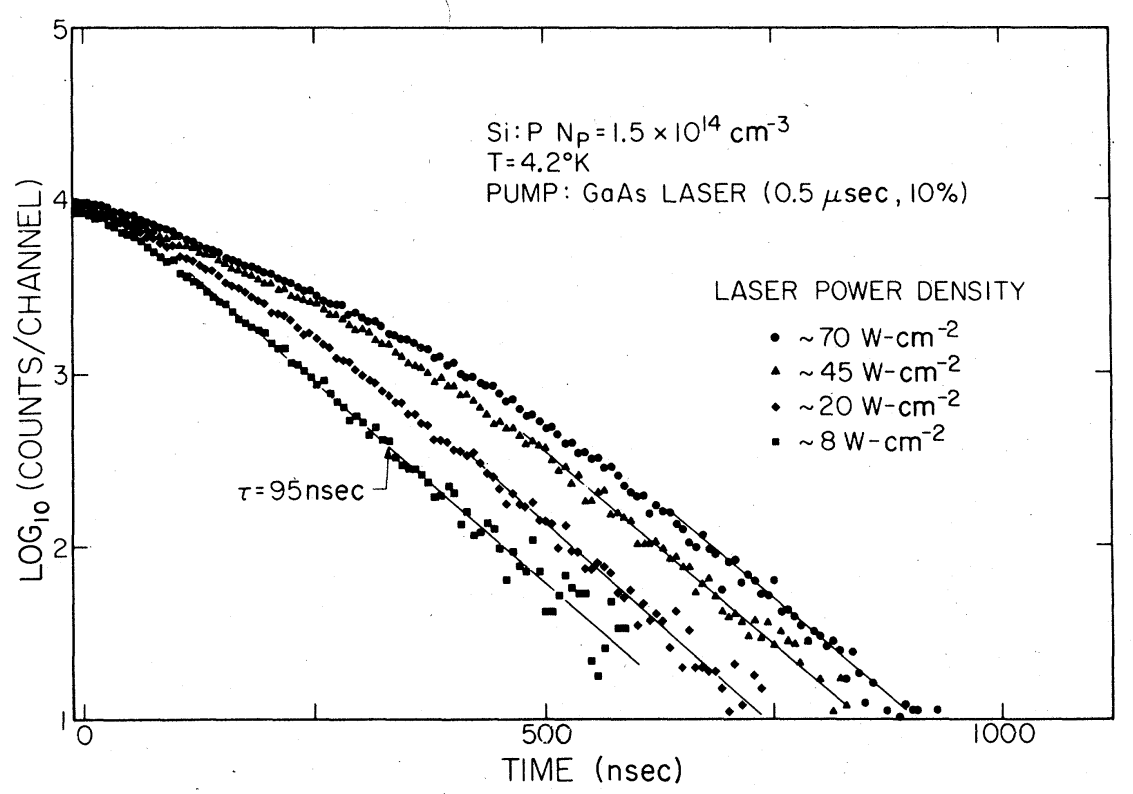

FIG. 3. Logarithm to the base ten of the transient decay of the $\alpha_{3}$ line in the no-phonon transition for a number of different laser pump power densities. The straight lines all have a slope appropriate to a 95 -nsec exponential decay time. 
ting an exponential decay to earlier segments of the curves, and may explain why our results differ from the decay times quoted by Sauer.

Figures 4,5 , and 6 show the decays for $\beta_{1}, \beta_{2}$, and $\beta_{3}$, respectively. In each figure, the points show the $\log$ of the number of counts/channel versus channel number (or time). Only one out of every three data points is plotted, to make the lines plotted on the figure visible. (All the data collected was used for the analysis, however.) The lines are "fits" made by adding other transient curves together, which will be discussed later. The lines are offset, and data repeated, to make comparison of the various fits easier.

The decay curve for $\beta_{1}$ (Fig. 4) shows three different regions. The first, just after the laser shuts off at $t=0$, shows a rounding discussed earlier. The fairly straight region from about 300 to $600 \mathrm{nsec}$ decays somewhat faster than that of the phosphorous bound exciton, clearly indicating that the $\beta_{1}$ line, without the $\alpha_{1}$ and other lines underneath it, has a decay time faster than $245 \mathrm{nsec}$. The last segment of the curve shows a definite break to a decay time much slower than that of phosphorus bound exciton, and is due to the boron bound exciton, with a lifetime of about 1000 nsec. $^{21}$
The decay curve for $\beta_{2}$ (Fig. 5) also has three dif ferent regions. First, it shows the same rounded pa as for all the other curves. The second part, from about 400 to $600 \mathrm{nsec}$, is again almost straight. The last segment of the curve, which breaks to a slower decay time, is due to the tail of a longer-lived line that overlaps $\beta_{2}$. This is probably either the phosphorus $\mathrm{BE}$, or a boron exciton-complex line. The $\mathrm{i}$ : itial part of this curve clearly decays faster than $\alpha_{2}$ even without subtracting out the longer-lived tail under the $\beta_{2}$ line.

The $\beta_{3}$ curve is shown in Fig. 6. The rounding o the initial part of the curve is not as apparent here : it was for $\beta_{1}$ and $\beta_{2}$, but it is still present. The dec is almost exponential until $\sim 500 \mathrm{nsec}$ after the lase shuts off, when the curve shows a definite break to longer decay time. Since $\beta_{3}$ sits on the shoulder of the TO-phonon $\alpha_{2}$ line, the $\alpha_{2}$ line causes at least part of the break. However, there is also a boron exciton-complex line at almost the same wavelength as $\alpha_{2},{ }^{3}$ which could also contribute to the break. T1 initial 200 or 300 nsec segment of the $\beta_{3}$ decay curv clearly decays faster than the NP $\alpha_{3}$, even before subtracting out the longer-lived component under $\mathrm{t}$ l $\beta_{3}$ line. The initial part of the $\beta_{3}$ also decays very slightly faster than the NP $\alpha_{4}$ line.

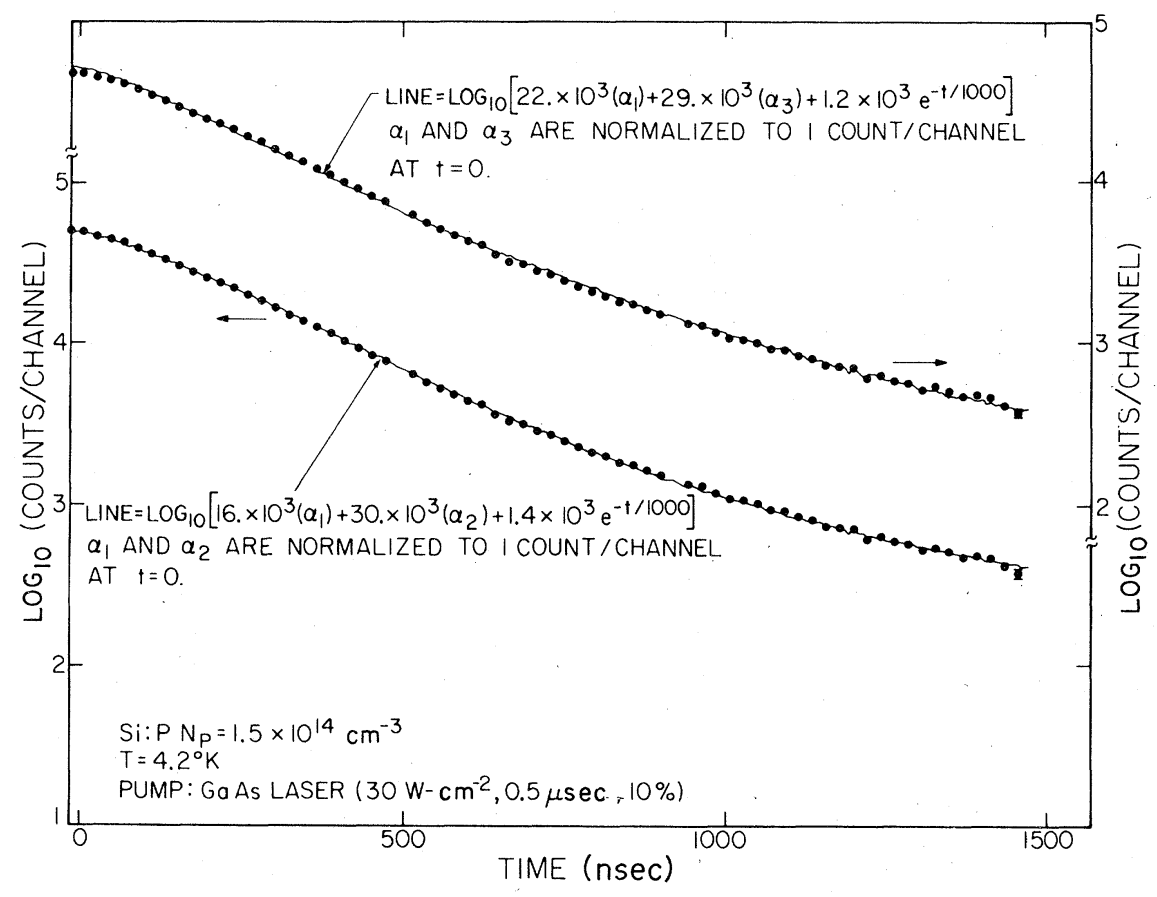

FIG. 4. Logarithm to the base ten of the transient decay of the $\beta_{1}$ line. The points are the experimental data which have been plotted twice with the upper set referring to the axis on the right and the lower set referring to the axis on the left. The solid lines are the results of fitting the experimental data to the indicated combinations of the decay of other lines. 


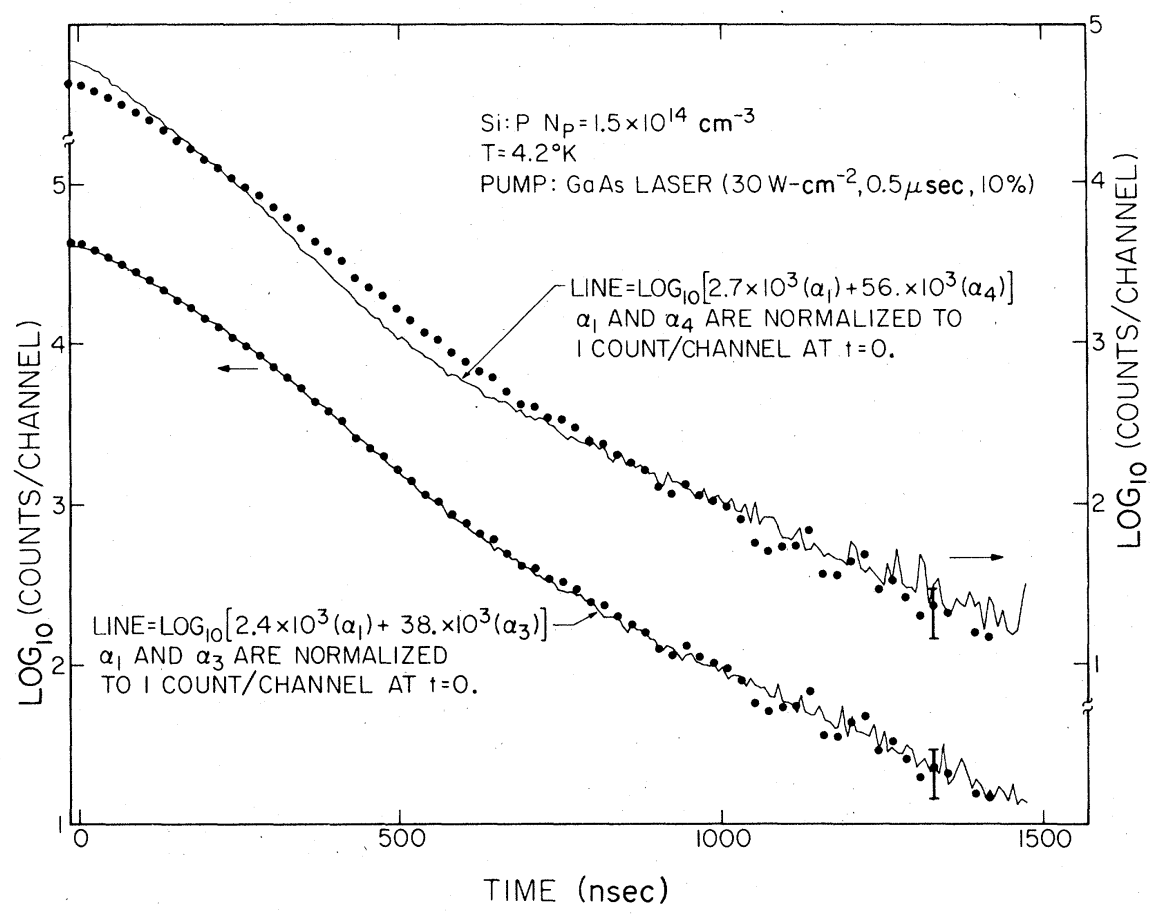

FIG. 5. Logarithm to the base ten of the transient decay of the $\beta_{2}$ line. The points are the experimental data which have been plotted twice with the upper set referring to the axis on the right and the lower set referring to the axis on the left. The solid lines are the results of fitting the experimental data to the indicated combinations of the decay of other lines.

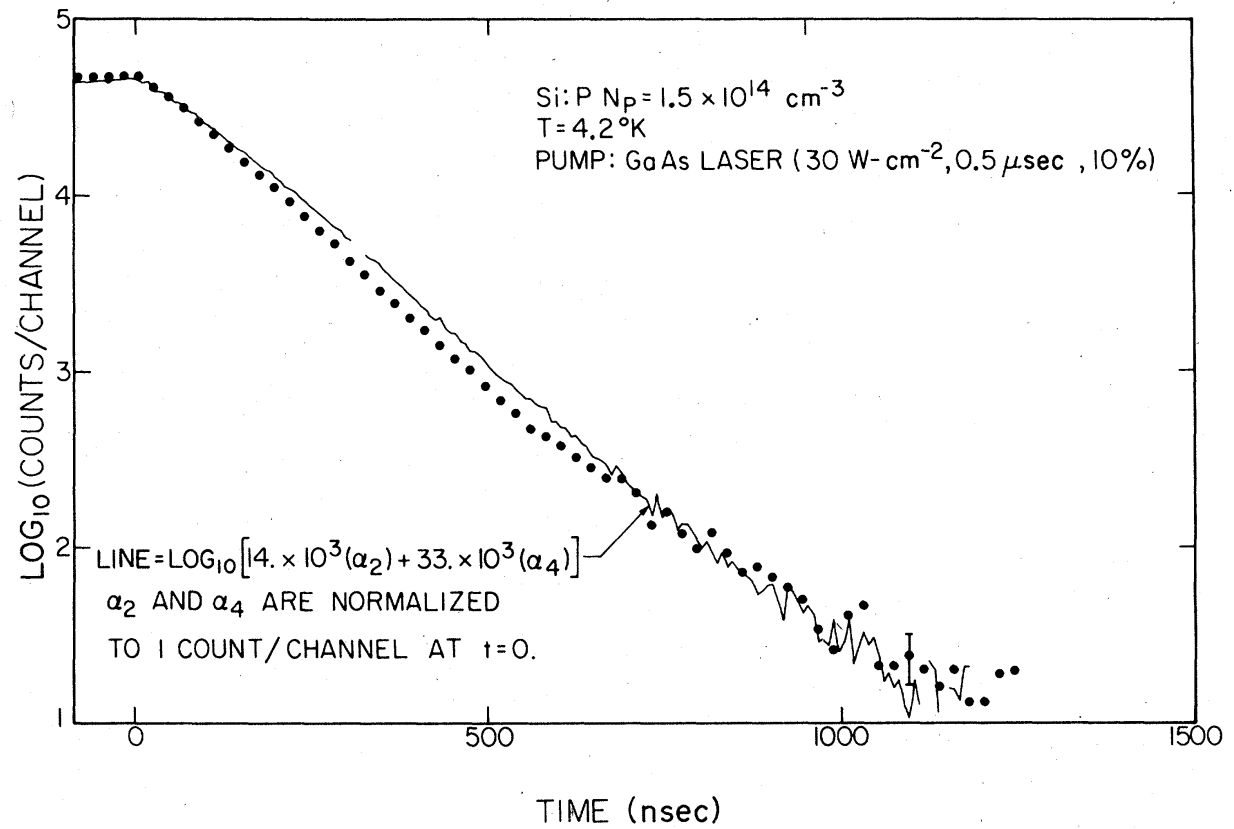

FIG. 6. Logarithm to the base ten of the transient decay of the $\beta_{3}$ line. The points are the experimental data. The solid line is the result of fitting the experimental data to the indicated combinations of the decay of other lines. 


\section{ANALYSIS OF DECAYS AND CONCLUSIONS}

The method of analysis differed for the $\alpha$ and $\beta$ series of decay curves. No other lines of appreciable intensity overlap the NP $\alpha_{1}, \alpha_{2}, \alpha_{3}$, or $\alpha_{4}$, and their decays reflect this by showing exponential behavior after some initial rounding. Therefore, the straightforward analysis of these transients, interpreting them each as the decay of a single luminescence line, is fairly reliable. This is not the case for the $\beta$ series of lines. The $\beta$ series is not visible in the NP spectrum, and the TO phonon replica of each of the three $\beta$ series lines overlaps an $\alpha$ series line. Also, the presence of boron bound-exciton lines and the possible presence of a broad background in the TO phonon spectrum further complicates the decays of the $\beta$ lines. Hence, the straightforward analysis used for the $\alpha$ lines is not appropriate for the $\beta$ lines.

To deal with the background and overlaps for the $\beta_{1}, \beta_{2}$, and $\beta_{3}$, various combinations of other decay curves were added together in an attempt to reproduce the particular $\beta$ decay under consideration. The actual data was used in these composite curves, because the rounding exhibited by all the decays ruled out fitting the $\beta$ curves analytically as the sum of exponentials. The amplitude of each component in the composite curve was determined by a nonlinear least-squares method. In plotting the resulting fit (see Figs. 4, 5, and 6) continuous curves were used to distinguish the fit from the $\beta$ decay data points.

The extraneous components of the $\beta_{1}$ decay were taken to come from the $\alpha_{1}$ line, since the $\beta_{1}$ sits on the shoulder of the TO bound exciton, and from the boron bound-exciton line, since this line is known to sit at approximately the same wavelength as $\beta_{1}$. The decay of the $\beta_{1}$ was compared with both the $\alpha_{2}$ and $\alpha_{3}$ lines. In each case, the extraneous components were added to the $\alpha$ data, and the result compared to $\beta_{1}$. More specifically, if $\beta_{1}(I), \alpha_{1}(I)$, etc., are the number of counts (minus dark count and afterpulsing) in channel $I$ on the MCA [corresponding to intensity of luminscence light detected during a time window at time $t(I)]$ then the function

$$
\begin{aligned}
\sum_{I} \mid \log _{10} \beta_{1}(I)-\log _{10}\left[a \alpha_{1}(I)+b \alpha_{i}(I)\right. \\
\left.+c e^{-t(I) / 1000 \mathrm{nsec}}\right]\left.\right|^{2},
\end{aligned}
$$

was minimized by varying the parameters $a, b$, and $c$. An analytic form was chosen to represent the effect of the boron, since the boron-BE transient curve itself could not be obtained for this sample. Figure 4 shows the fits. The upper curve is the result using $\alpha_{i}=\alpha_{3}$ in Eq. (4.1). The points are the $\log$ of the $\beta_{1}$ data, the line is the $\log$ of the sum of parts. The fit looks fairly close; it may, however, decay too steeply at the beginning to fit the data.
The second curve is the result for $i=2$. This fit is slightly better than the previous one, as indicated by the error criterion of the least-squares fitting procedure. However, the similarity of the two different fits indicates that this is not a particularly sensitive test for the $\beta_{1}$ decay time.

The extraneous component of the $\beta_{2}$ transient was taken to come from a tail of the $\alpha_{1}$. The function to be minimized for the fit to this decay was

$$
\sum_{I}\left|\log _{10} \beta_{2}(I)-\log _{10}\left[a \alpha_{1}(I)+b \alpha_{i}(I)\right]\right|^{2},
$$

done by varying $a$ and $b$. Figure 5 shows the results. The points are the $\log$ of the $\beta_{2}$ data, the line the log of the fit, drawn as a continuous curve. The top plot in the figure is for $i=4$ in Eq. (4.2), clearly a bad fit. The bottom plot is for $i=3$, and seems to be an adequate fit. However, it is not clear that $\alpha_{1}$ is the only line that could affect the $\beta_{2}$ decay. A boron multiplebound-exciton line sits between the $\beta_{2}$ and $\alpha_{1}$, as does the LO-phonon replica of $\alpha_{2}$.

We attempted to fit the $\beta_{3}$ decay to the $\alpha_{4}$ decay. Overlap from the $\alpha_{2}$ line was included by adding the $\alpha_{2}$ transient to the $\alpha_{4}$ transient, the coefficients of each determined by minimizing

$$
\sum_{I}\left|\log _{10} \beta_{3}(I)-\log _{10}\left[a \alpha_{2}(I)+b \alpha_{4}(I)\right]\right|^{2} .
$$

This fit, shown as a solid line in Fig. 6, is not a particularly good one. As pointed out earlier, $\beta_{3}$ decays slightly faster than the NP $\alpha_{4}$ line at early times even before subtracting out the longer-lived component under $\beta_{3}$. However, this still does not rule out the possibility that $\beta_{3}$ decays like $\alpha_{4}$, because the broad background found in the TO-phonon spectrum has not been taken into account. A comparison of the transient curves of the NP and TO-phonon replicas of the $\alpha_{3}$ line indicates the broad background decays faster than $\alpha_{3}$, because the TO-phonon replica of $\alpha_{3}$ decays slightly faster at early times than its NP counterpart. If this same background were present under the $\beta_{3}$ line, it alone could cause the fitting procedure to fail, because the background was not included in the composite curve.

The data and analysis presented above allow us to place limits on the decay of the $\beta$-series lines, but do not allow us to conclude unambiguously whether or not a given $\beta$ decay is identical to any of the $\alpha$ decays. The $\beta_{1}$ line decays faster than $\alpha_{1}$, but its decay may be like that of either $\alpha_{2}$ or $\alpha_{3}$. The error criterion of the fitting procedure indicates $\alpha_{2}$ provides a better fit, but the $\alpha_{3}$ fit is fairly good showing that the fitting procedure is not a very sensitive test of the $\beta_{1}$ decay. Further, our uncertainty in the precise form of the decay of the boron BE, particularly at early times, is comparable to the difference in the fit for $\alpha_{2}$ and $\alpha_{3}$. The limits on the $\beta_{2}$ decay are better de- 
fined. The $\beta_{2}$ line decays faster than $\alpha_{2}$, but more slowly than $\alpha_{4}$. The fit with the $\alpha_{3}$ decay works well. Finally the $\beta_{3}$ decays more rapidly than the $\alpha_{3}$. The fit to the $\alpha_{4}$ is not very good; however, the differences could be due to an unaccounted for background. These results do not settle conclusively the question of the validity of the assignments given in the shell model, although the results are consistent with it.

\section{ACKNOWLEDGMENTS}

The authors wish to acknowledge helpful conversations with Ken Elliott and Gordon Mitchard. The transmutation doping of the silicon sample was carried out by Professor R. Hart of Texas A\&M University with the assistance of M. Young of Hughes Research Laboratories. This research was supported in part by ONR and ARPA.
"Present address: Dept. of Electrical Engineering, Princeton Univ. Princeton, New Jersey 08540.

${ }^{1}$ A. S. Kaminskii, Y. E. Pokrovskii, and N. V. Alkeev, Zh. Eksp. Teor. Fiz. 59, 1937 (1970).

${ }^{2}$ R. Sauer, Phys. Rev. Lett. 31, 376 (1973).

${ }^{3}$ K. Kosai and M. Gershenzon, Phys. Rev. B 9, 723 (1974).

${ }^{4}$ P. J. Dean, D. Herbert, D. Bimberg, and W. J. Choyke, Phys. Rev. Lett. 37, 1635 (1976).

${ }^{5}$ M. L. W. Thewalt, Phys. Rev. Lett. 38, 521 (1977).

${ }^{6}$ S. A. Lyon, D. L. Smith, and T. C. McGill, Phys. Rev. B 17, 2620 (1977).

${ }^{7}$ M. L. W. Thewalt, Solid State Commun. 21, 937 (1977).

${ }^{8}$ M. L. W. Thewalt, Can. J. Phys. 55, 1463 (1977).

${ }^{9}$ R. Sauer, W. Schmid, and J. Weber, Solid State Commun. 24, 507 (1977).

${ }^{10} \mathrm{M}$. L. W. Thewalt, Solid State Commun. 25, 513 (1978).

${ }^{11}$ S. A. Lyon, D. L. Smith, and T. C. McGill, Phys. Rev. Lett. 41,56 (1978).

${ }^{12}$ M. L. W. Thewalt and J. A. Rostworowski, Phys. Rev. Lett. $\underline{41}, 808$ (1978).
${ }^{13}$ K. R. Elliott and T. C. McGill, Solid State Commun. 28 , 491 (1978).

${ }^{14} \mathrm{~T}$. N. Morgan, in Proceedings of XIII International Conference on the Physics of Semiconductors, edited by F. G. Fumi (Marves, Rome, 1977), p. 825.

${ }^{15} \mathrm{G}$. Kirczenow, Solid State Commun. 21713 (1977).

${ }^{16} \mathrm{G}$. Kirczenow, Can. J. Phys. 55, 1787 (1977).

${ }^{17} \mathrm{The}$ three lowest levels of the donor in $\mathrm{Si}$ are those functions made up of an envelope function with $S$-like symmetry and $\Gamma_{1}, \Gamma_{3}$, and $\Gamma_{5}$ combinations of the states of various valleys. See, for example, F. Bassani and G. Pastori Parravicini, Electronic States and Optical Transitions in Solids (Pergamon, Oxford, 1975), Chap. 7, Sec. 3.

${ }^{18}$ M. L. W. Thewalt, in Physics of Semiconductors, 1978, edited by B. L. H. Wilson, IOP Conf. Ser. No. 43 (IPPS, London, 1979).

${ }^{19}$ S. A. Lyon (unpublished).

${ }^{20}$ M. Tajima Appl. Phys. Lett. 32, 719 (1978).

${ }^{21}$ S. A. Lyon, G. C. Osbourn, D. L. Smith, and T. C. McGill, Solid State Commun. 23, 425 (1977). 